The Astrophysical Journal, 270:L41-L42, 1983 July 15

( 1983 . The American Astronomical Society. All rights reserved. Printed in U.S.A.

\title{
THE VELOCITY DISPERSION OF THE GLOBULAR CLUSTERS IN THE FORNAX DWARF GALAXY
}

\author{
Judith G. COHEN \\ California Institute of Technology \\ Received 1982 December 28; accepted 1983 March 28
}

\begin{abstract}
Observations of the four brightest of the globular clusters in the Fornax dwarf spheroidal galaxy yield radial velocities whose accuracy is $\pm 10 \mathrm{~km} \mathrm{~s}^{-1}$. The observed velocity dispersion for the Fornax galaxy from this sample is too small to be detected $\left(\sigma=6 \mathrm{~km} \mathrm{~s}^{-1}\right)$. Thus, at the $95 \%$ confidence level, $M / L_{v}$ is less than 8.
\end{abstract}

Subject headings: clusters: globular - galaxies: internal motions

\section{MOTIVATION AND OBSERVATIONS}

Aaronson (1983) recently claimed to determine a velocity dispersion in the Draco dwarf galaxy of 9 $\mathrm{km} \mathrm{s}^{-1}$ from radial velocities of three carbon stars measured with an accuracy of $\pm 1 \mathrm{~km} \mathrm{~s}^{-1}$. From this he inferred the existence of a high mass-to-light ratio in Draco, characteristic of that for the dark matter presumed to exist in the halo of our own Galaxy. Faber and Lin (1983) predicted a velocity dispersion of $34 \mathrm{~km} \mathrm{~s}^{-1}$ in the more luminous Fornax dwarf galaxy assuming a similar, large mass-to-light ratio. Radial velocities for three of the globular clusters in Fornax have been compiled by Webbink (1981) but are not sufficiently accurate to test this assertion. We therefore observed the four brightest of the clusters identified by Hodge (1961) in Fornax on 1982 September 15 and September 19 at the du Pont telescope of the Las Campanas Observatory using the intensified reticon detector constructed by $\mathrm{S}$. Shectman. Slits $2^{\prime \prime}$ wide and $8^{\prime \prime}$ high were used; the spectra are fully sky subtracted. Comparison arcs were taken before and after each spectrum. Typical exposure times were 45 minutes except for the faintest cluster (G2), where integration times of 1 hour or more were necessary. All four clusters were observed on each night, while on September 15, the brightest cluster (G3) was observed twice. Over the full array of 3700 pixels, more than 750,000 counts were recorded, except for cluster G2 where only 100,000 to $2,000,000$ counts were obtained. The dispersion was fitted by a fifth-order polynomial to 25 comparison lines and was roughly $0.59 \AA$ per pixel, while the full width at half-maximum of comparison lines is 3 pixels. The wavelength region covered was 4240-6000 $\AA$.

\section{ANALYSIS AND DISCUSSION}

The most prominent spectral features are the $\mathrm{Mg}$ triplet (but this and the other metal lines are very weak) and the Balmer lines. Thus only the interval $4250-5500$ $\AA$ was used in the cross correlation. As it was not clear what to use as a spectral template and the goal was the determination of the velocity dispersion, the spectra from each night were cross-correlated using software written by P. J. Young with each of the three brightest clusters in turn serving as templates. The mean of these three relative radial velocities is listed in Table 1 for each of the two nights. The typical uncertainty in the radial velocities is $\pm 10 \mathrm{~km} \mathrm{~s}^{-1}$, except for the faintest cluster G2, where the uncertainty is $\pm 20 \mathrm{~km} \mathrm{~s}^{-1}$. (This is consistent with cross-correlations accurate to onefourth of a pixel for the higher precision spectra.)

The mean relative velocities for the two nights are given in the final column of Table 1 . The velocity dispersion of these four clusters is $6 \mathrm{~km} \mathrm{~s}^{-1}$, which reflects entirely the uncertainties in the data. There is no evidence from this data for the predicted large-velocity dispersion in the Fornax dwarf galaxy. The $\chi^{2}$ test indicates that, ignoring observational errors and including all four clusters, there is less than a $5 \%$ chance that the velocity dispersion is greater than $18 \mathrm{~km} \mathrm{~s}^{-1}$. This must be considered a most generous estimate of the maximum velocity dispersion. Thus, at the $95 \%$ confidence level, $M / L_{v}$ in Fornax is less than 8 , a value closer to that of the globular clusters. (The dynamically determined $M / L$ ratio for $\mathrm{M} 3$ is $2.5 \pm 0.5$ [Gunn and Griffin 1979], with $M / L_{v} \approx 80 \%$ of $M / L$ for metal-poor globular clusters.)

The absolute radial velocities can be determined by using the spectra of several Small Magellanic Cloud (SMC) clusters taken immediately prior to those of the Fornax globular clusters on 1982 September 15 with the same instrumental configuration. NGC 121, a bona fide old globular cluster in the SMC (see Searle, Wilkinson, and Bagnuolo 1980), has a spectrum quite comparable to that of the program objects. Its radial velocity is $154 \pm 25 \mathrm{~km} \mathrm{~s}^{-1}$ (Searle and Smith 1983). Since this is a 
TABLE 1

Relative Radial Velocities of THE Fornax Globular CluSTERS

\begin{tabular}{cccc}
\hline \hline Object & $\begin{array}{c}\text { Sep 15 } \\
\left(\mathrm{km} \mathrm{s}^{-1}\right)\end{array}$ & $\begin{array}{c}\text { Sep 19 } \\
\left(\mathrm{km} \mathrm{s}^{-1}\right)\end{array}$ & $\begin{array}{c}\text { Mean } \\
\left(\mathrm{km} \mathrm{s}^{-1}\right)\end{array}$ \\
\hline G2 $\ldots \ldots \ldots$ & -18.5 & +36.7 & +9.1 \\
G3 $\ldots \ldots \ldots$ & -7.4 & +10.1 & +0.9 \\
G3 $\ldots \ldots \ldots$ & $\ldots$ & +8.3 & +11.7 \\
G4 $\ldots \ldots \ldots$ & +0.4 & -11.7 & -5.7 \\
G5 $\ldots \ldots \ldots$ & +6.8 & +2.5 & +4.7 \\
\hline
\end{tabular}

possible halo cluster and the motion of the gas in the SMC is somewhat chaotic, one cannot use the $21 \mathrm{~cm}$ radial velocity in that area of the SMC $(130 \pm 10$ $\mathrm{km} \mathrm{s}^{-1}$, Hindman and Balnaves 1967) to refine the optical velocity for the cluster. Via cross-correlations, we find that the mean of the three brightest globular clusters in the Fornax dwarf galaxy have a radial mean velocity of $-88 \mathrm{~km} \mathrm{~s}^{-1}$ with respect to NGC 121, or $V_{r}=66 \pm 25 \mathrm{~km} \mathrm{~s}^{-1}$.

Aaronson's claim of a large velocity dispersion for Draco suffers from the possible problems of membership in binaries and inadequate statistics (only one observation of two of the three objects). If his claim should be subsequently substantiated, it is more likely that this reflects the disruption of the Draco galaxy, predicted by Hodge and Michie (1969), rather than the presence of a large mass of dark material.

I am grateful to the director of the Mount Wilson and Las Campanas Observatories for a generous allowance of telescope time, to the NSF fund for travel to foreign observatories, and to David Tytler for advice on statistics.

\section{REFERENCES}

Aaronson, M. 1983, Ap. J. (Letters), 266, L11.

Faber, S. M., and Lin, D. C. 1983, Ap. J., 266, L17.

Gunn, J. E., and Griffin, R. F. 1979, A.J., 84, 752.

Hindman, J. V., and Balnaves, K. M. 1967, Australian J. Phys., Ap. Suppl., 4.

Hodge, P. W. 1961, A.J., 66, 83.
Hodge, P. W., and Michie, R. W. 1969, A.J., 76, 587.

Searle, L., and Smith, H. 1983, private communication.

Searle, L., Wilkinson, A., and Bagnuolo, W. G. 1980, Ap. J., 239, 803.

Webbink, R. F. 1981, Ap. J. Suppl., 45, 259.

Judith G. Cohen: 105-24, California Institute of Technology, Pasadena, CA 91125 